\title{
ON THE EXISTENCE OF UNRAMIFIED SEPARABLE INFINITE SOLVABLE EXTENSIONS OF FUNCTION FIELDS OVER FINITE FIELDS*
}

\author{
HISASI MORIKAWA
}

In the present note, using the results in the previous paper, ${ }^{1)}$ we shall prove the following existence theorem:

Theorem. Let $k$ be a finite field with $q$ elements and $K / k$ be a regular extension of dimension one over $k$. Then, if $q \geqslant 11$ and the genus $g_{k}$ of $K_{/}^{\prime} k$ is greater than one, there exists an unramified separable infinite solvable extension of $K$ which is regular over $k^{2}{ }^{2}$

\section{$\S 1$. The results in [1]}

1.1. Let $k$ be a finite field with $q$ elements and $K / k$ be a regular extension of dimension one over $k$. Let $L / k$ be an unramified separable normal extension of $K$ which is also regular over $k$. We denote by $G(L / K)$ the galois group of $L / K$. We denote by $C_{L}$ and $C_{K}$ non-singular complete models of $K / k$ and $L_{/} / k$, respectively, and denote by $\hat{\pi}_{L / K}$ the trace mapping of $C_{L}$ onto $C_{K}$. We denote by $J_{L}\left(J_{K}\right)$ and $\varphi_{L}\left(\varphi_{K}\right)$ the jacobian variety of $C_{L}\left(C_{K}\right)$ and a canonical mapping of $C_{L}\left(C_{K}\right)$ into $J_{L}\left(J_{K}\right)$, respectively, where we may assume that $J_{L}\left(J_{K}\right)$ and $\varphi_{L}\left(\varphi_{K}\right)$ are also defined over $k$. We denote by $\pi_{L / K}$ the extension of $\hat{\pi}_{L / K}$ which is a homomorphism of $J_{L}$ onto $J_{K}$ such that $\pi_{L / K} \circ \varphi_{L}=\varphi_{K} \circ \hat{\pi}_{L / K}+c$ with a constant point $c$. After a suitable translation of $\varsigma_{k}$, we assume that

$$
\pi_{L^{\prime} K} \circ \varphi_{L}=\varphi_{K} \circ \hat{\pi}_{L^{\prime} K} .
$$

We denote by $A(, k)$ the subgroup of $k$-rational points of a commutative group variety $A$.

Each element $\varepsilon_{\text {, of }} G\left(L_{L} / K\right)$ induces an automorphism $\left\{\eta_{L}\left(\varepsilon_{\nu}\right)\right\}$ of $J_{L}$ and

Received October 25, 1957.

* This note was prepared while the author was a Yukawa Fellow at Osaka University.

1) We shall refer this paper with [1].

2) We mean by an infinite solvable extension a solvable extension of infinite degree. 
a translation $\left\{a_{L}\left(\hat{\varepsilon}_{\nu}\right)\right\}$ on $J_{L}$, both defined over $k$, such that

$$
\varphi_{L}\left(P^{\varepsilon_{2}-1}\right)=\eta_{L}\left(\varepsilon_{2}\right) \varphi_{L}(P)+a_{L}\left(\varepsilon_{\nu}\right) \quad\left(\varepsilon_{\nu} \in G(L / K), P \in C_{L}\right),
$$

where $\eta_{L}\left(\varepsilon_{2}\right)$ and $a_{L}\left(\varepsilon_{\nu}\right) \quad\left(\varepsilon_{\nu} \in G(L / K)\right)$ are defined over $k$ and they are independent on the choice of $P$. From the definition we have

$$
a_{L}\left(\varepsilon_{,} \varepsilon_{j}\right)=\eta_{L}\left(\varepsilon_{\nu}\right) a_{L}\left(\varepsilon_{\mu}\right)+a_{L}\left(\varepsilon_{\nu}\right) .
$$

If we put $\varphi_{L}^{\prime}=c_{L}+c$ and $\varphi_{L}^{\prime}\left(P^{\varepsilon_{\nu}-1}\right)=\eta_{L}\left(\varepsilon_{0}\right) \varphi_{L}^{\prime}(P)+a_{L}^{\prime}\left(\varepsilon_{\nu}\right)$, then we have

$$
a_{L}^{\prime}\left(\varepsilon_{\nu}\right)=a_{L}\left(\varepsilon_{\nu}\right)+\left(\delta_{J_{L}}-\gamma_{L}\left(\varepsilon_{\nu}\right)\right) c .
$$

1. 2. Let $x$ be a generic point of $J_{L}$ over $k$. Let $A_{L / K}$ and $B_{I / K}$ be respectively the loci of

$$
\left(\sum_{\varepsilon_{2} \in G(L / K)} \gamma_{\gamma_{L}}\left(\varepsilon_{.,}\right)\right) x \text { and }\left(\sum_{\varepsilon, \in(i, L / K)}\left(\delta_{J_{L}}-\eta_{L_{L}}\left(\varepsilon_{\nu}\right)\right)\right) x^{3)}
$$

over $k$. Let $\bar{A}_{L / K}$ be the quotient abelian variety of $J_{L}$ by $B_{L / K}$ and $\alpha_{L / K}$ be the natural mapping of $J_{L}$ onto $\bar{A}_{L / K}$. Then $A_{L / K}, B_{L / K}, \bar{A}_{L / K}$ and $\alpha_{L / K}$ are defined over k. $A_{L / K}$ and $B_{L / K}$ generate $J_{L}$ and the intersection $A_{L / K} \cap B_{L / K}$ is a finite group. Moreover $B_{L / K}$ is the irreducible component of $\pi_{L / K}^{-1}(0)$. Let $\bar{\pi}_{L / K}$ be the homomorphism of $\bar{A}_{L / K}$ onto $J_{K}$ such that

$$
\pi_{L / K}=\bar{\pi}_{L / K} \alpha_{L / K} .
$$

In [1] we have proved the following facts:

TheOREM A. $\bar{\pi}_{L / K}$ is separable and $\bar{\pi}_{L / K}^{-1}(0)=\left\{\alpha_{L / K} a_{L}\left(\varepsilon_{\nu}\right) \mid \varepsilon_{\nu} \in G(L / K)\right\}$. If $L / K$ is an unramified separable abelian extension, then the mapping $\varepsilon_{\nu} \rightarrow \alpha_{L / K} a_{L}\left(\varepsilon_{v}\right)$ is an isomorphism of $G(L / K)$ onto $\bar{\pi}_{L / K}^{-1}(0)$.

THEOREM B. If $L / K$ is an unramified separable abelian extension, then

$$
J_{K}(, k) / \pi_{L / K}\left(J_{L}(, k)\right) \cong G(L / K) .
$$

Theorem C. Let $g$ be any subgroup of $J_{k}(, k)$. Then there exists an unramified separable abelian extension $K(g)$ of $K$ such that $\mathrm{i}) K(\mathfrak{g})$ is regular over $k$ and ii) $\pi_{\kappa(\mathfrak{g}) ! K} J_{L(\mathfrak{g})}(, k)=\mathfrak{g}$.

The field $K(g)$ in theorem $C$ is given as follows:

Let $A$ be the quotient abelian variety of $J_{K}$ by $g$ and $\mu$ be the natural homomorphism of $J_{K}$ onto 1 . Let $\mathfrak{p}_{J_{K}}$ be the endomorphism of $J_{K}$ which is

3) $\delta_{J}$ means the identity endomorphism of $J$. 
induced by the automorphism $\xi \rightarrow \xi^{q}$ of the universal domain and $\lambda$ be the homomorphism of $A$ onto $J_{K}$ such that $\lambda \mu=\delta_{J_{K}}-p_{J_{K}}$. Then $\lambda$ and $\mu$ are separable homomorphisms defined over $k$. Let $y$ be the poin' of $A$ such that $k(\lambda y)=K$ and $\lambda y=\varphi_{K}(P)$ with a point $P$ of $C_{K}$. Then the field $K(g)$ is $k(y)$ and the galois automorphisms of $K(\mathrm{~g}) / K$ are induced by the translations $y \rightarrow y+t$ $\left(t \in \lambda^{-1}(0)\right)$.

\section{§. The proof of the theorem}

2. 1. To prove the theorem, it is sufficient to prove the following two lemmas :

Lemma 1. Let $L / K$ be an unramified separable normal extension which is also regular over $k$ and $g$ be a subgroup of $J_{L}(, k)$. Then $L(g)$ is normal over $K$ if and only if the following conditions are satisfied for every $\varepsilon_{\nu} \in G(L / K)$ :

i) $\eta_{L}\left(\varepsilon_{\nu}\right)(g)=g$,

ii) $a_{L}\left(\varepsilon_{\nu}\right) \in g$.

Lemma 2. Let $L / K$ be an unramified separable normal extension which is also regular over $k$ and $l$ be a prime number. Let is be a subgroup of $J_{L}(, k)$ such that $L(g) / K$ is normal and $[L(g): L]=l$. Then if $q \geq 11$ and the genus $g_{K}$ of $K / k$ is greater than one there exists a subgroup $\mathfrak{s}_{1}$ of $J_{L / G)}(, k)$ such that i) $g_{1} \neq J_{L(g)}(, k)$ and ii) $(L(g))\left(g_{1}\right)$ is normal over $K$.

2. 2. The proof of lemma 1 .

First we assume that $L(g) / K$ is normal and denote by $\left[\varepsilon_{3}\right]$ a representative of $\varepsilon_{\nu} \in G(L / K)$ in $G(L(\mathfrak{g}) / K)$. Then we have i) $\eta_{L}\left(\varepsilon_{\nu}\right) \pi_{L / \mathcal{G}) / L}=\pi_{L(\mathcal{G}) / L} \eta_{L(\mathcal{G})}\left(\left[\varepsilon_{\nu}\right]\right)$ and ii) $a_{L}\left(\varepsilon_{\nu}\right)=\pi_{L(\mathfrak{G}) ! L} a_{L(\mathfrak{g})}\left(\left[\varepsilon_{\nu}\right]\right) \quad\left(\varepsilon_{\nu} \in G(L / K)\right)$. Hence, by virtue of theorem $C$, we have

$$
\text { i) } \begin{aligned}
a_{L}\left(\varepsilon_{\nu}\right) & \left.=\pi_{L(\mathfrak{g}) / L} a_{L(\mathfrak{g})}\left(\left[\varepsilon_{\nu}\right]\right) \text { and ii }\right) \eta_{L}\left(\varepsilon_{\nu}\right)(g)=\eta_{L}\left(\varepsilon_{\nu}\right)\left(\pi_{L /(\mathfrak{g}) / L} J_{L(\mathfrak{g})}(, k)\right) \\
& =\pi_{L(\mathfrak{g}) / L} \eta_{L(\mathfrak{g})}\left(\left[\varepsilon_{\nu}\right]\right) J_{L(\mathfrak{g})}(, k)=\pi_{L(\mathfrak{g}) / L} J_{L(\mathfrak{g})}(, k)=\mathfrak{g} \quad\left(\varepsilon_{\nu} \in G(L / K)\right) .
\end{aligned}
$$

Conversely we assume that 8 satisfies the conditions of the lemma. Let $y$ be a point of $\bar{A}_{L(\mathcal{G}) / L}$ such that $k\left(\bar{\pi}_{L(\mathcal{G}) / L} y\right)=L$ and $\bar{\pi}_{L(\mathcal{G}) / L} y$ is a point of $\varphi_{L}\left(C_{L}\right)$. By virtue of theorem $C, \bar{A}_{L(g) / L}$ is the quotient variety of $J_{L}$ by $g$ and $\mu$ is the natural mapping of $J_{L}$ onto $\bar{A}_{L(\mathcal{g}) / L}$, where $\mu$ is the homomorphism of $J_{L}$ onto $\bar{A}_{L(\mathfrak{g}) / L}$ such that $\pi_{L(\mathfrak{g}) / L} \mu=\delta_{J_{K}}-\mathfrak{p}_{J_{K}}$. Namely $k(y)=L(\mathfrak{g})$. Since $a_{L}\left(\varepsilon_{\nu}\right) \in \mathfrak{g}$, 
there exist points $b\left(\left[\varepsilon_{\nu}\right]\right)$ in $\bar{A}_{L(g) / L}(, k)$ such that $\bar{\pi}_{L(g) / L} b\left(\left[\varepsilon_{\nu}\right]\right)=a_{L}\left(\varepsilon_{\nu}\right)$ $\left(\varepsilon_{,} \in G(L / K)\right)$. From the condition i) there exist automorphisms $\eta\left(\left[\varepsilon_{v}\right]\right)$ of $\bar{A}_{L(\mathfrak{g}) / L}$ such that $\bar{\pi}_{L(\mathfrak{g}) / L} \eta\left(\left[\varepsilon_{\nu}\right]\right)=\eta_{L L}\left(\varepsilon_{\nu}\right) \bar{\pi}_{L(\mathfrak{g}) / L}\left(\varepsilon_{\nu} \in G(L / K)\right)$. Let $\tilde{C}$ be the locus of $y$ over $k$. Then $\eta\left(\left[\varepsilon_{v}\right]\right) y+b\left(\left[\varepsilon_{v}\right]\right)+t\left(\varepsilon_{v} \in G(L / K), t \in \bar{\pi}_{L / g) / L}^{-1}(0)\right)$ are also points on $\tilde{C}$ and they are conjugates of $y$ over $K$. This proves that $L(g)$ is normal over $K$.

2.3. The proof of lemma 2

We denote by $\varepsilon$ the generator of $G(L(g) / L)$ and denote by $\left[\varepsilon_{\nu}\right]$ a representative of $\varepsilon_{\nu}$ in $G(L(\mathfrak{g}) / K)$. Since $(\varepsilon)$ is normal in $G(L(\mathfrak{g}) / K)$, there exists an integer $s_{\nu}$ such that $\left[\varepsilon_{\nu}\right] \varepsilon\left[\varepsilon_{\nu}\right]^{-1}=\varepsilon^{s_{\nu}}\left(\varepsilon_{\nu} \in G(L / K)\right)$.

Since $\left.\sum_{\nu=1}^{l}\left(\delta_{J_{L(\mathfrak{g})}}-\eta_{L(\mathfrak{g})}\left(\varepsilon^{\nu}\right)\right)=\delta_{J_{L}(\mathfrak{g})}-\eta_{L(\mathfrak{g})}(\varepsilon)\right)\left(\sum_{\nu=1}^{l}\left(\delta_{J_{L}(\mathfrak{g})}+\eta_{L(\mathfrak{g})}(\varepsilon)+\ldots\right.\right.$ $\left.+\eta_{L(\mathfrak{g})}\left(\varepsilon^{\nu-1}\right)\right)$, we observe that $B_{L(\mathfrak{g}) / L} \leqq\left(\delta_{J_{L(\mathfrak{g})}}-\eta_{L(\mathfrak{g})}(\varepsilon)\right)\left(J_{L(\mathfrak{g})}\right)$. On the other hand $J_{L(\mathfrak{g})}$ is generated by $B_{L(\mathfrak{g}) / L}$ and $A_{L(\mathfrak{g}) / L}$ and $\left(\delta_{J_{L / \mathfrak{g}}}-\eta_{L(\mathfrak{g})}(\varepsilon)\right)\left(A_{L(\mathfrak{g}) / L}\right)=0$, hence $B_{L(\mathfrak{g}) / L}=\left(\delta_{J_{L}(\mathfrak{g})}-\eta_{L(\mathcal{G})}(\varepsilon)\right)\left(J_{L(\mathfrak{g})}\right)$. Therefore, by virtue of (3), if we translate $\varphi_{L(\mathfrak{g})}$ suitably, we can assume that $a_{L(\mathfrak{g})}(\varepsilon)$ belongs to $A_{L(\mathfrak{g}) / L}$. From (2) we have

$$
\begin{aligned}
a_{L(\mathfrak{g})}\left(\varepsilon^{s_{\nu}}\right)= & a_{L(\mathfrak{g})}\left(\left[\varepsilon_{\nu}\right] \varepsilon\left[\varepsilon_{\nu}\right]^{-1}\right) \\
= & \eta_{L(\mathfrak{g})}\left(\left[\varepsilon_{\nu}\right]\right) \eta_{L(\mathfrak{g})}(\varepsilon) a_{L(\mathfrak{g})}\left(\left[\varepsilon_{\nu}\right]^{-1}\right)+\eta_{L(\mathfrak{g})}\left(\left[\varepsilon_{v}\right]\right) a_{L(\mathfrak{g})}(\varepsilon) \\
& +a_{L(\mathfrak{g})}\left(\left[\varepsilon_{\nu}\right]\right) \\
= & \eta_{L(\mathfrak{g})}\left(\left[\varepsilon_{\nu}\right]\right)\left(\eta_{L(\mathfrak{g})}(\varepsilon)-\delta_{J_{L(\mathfrak{g}}}\right) a_{L(\mathfrak{g})}\left(\left[\varepsilon_{\nu}\right]^{-1}\right) \\
& +\left(\eta_{L(\mathfrak{g})}\left(\left[\varepsilon_{\nu}\right]\right) a_{L(\mathfrak{g})}\left([\varepsilon]^{-1}\right)+a_{L(\mathfrak{g})}\left(\left[\varepsilon_{\nu}\right]\right)\right) \\
& +\eta_{L(\mathfrak{g})}\left(\left[\varepsilon_{\nu}\right]\right) a_{L(\mathfrak{g})}(\varepsilon) \\
= & \eta_{L(\mathfrak{g})}\left(\left[\varepsilon_{\nu}\right]\right)\left(\eta_{L(\mathfrak{g})}(\varepsilon)-\delta_{J_{L}(\mathfrak{g})}\right) a_{L(\mathfrak{g})}\left(\left[\varepsilon_{\nu}\right]^{-1}\right) \\
& +\eta_{L(\mathfrak{g})}\left(\left[\varepsilon_{\nu}\right]\right) a_{L(\mathfrak{g})}(\varepsilon) .
\end{aligned}
$$

On the other hand, since $\eta_{L(\mathfrak{g})}\left(\left[\varepsilon_{\nu}\right]\right)\left(\delta_{J_{L}(\mathfrak{g})}+\eta_{L(\mathfrak{g})}(\varepsilon)+\ldots+\eta_{L(\mathfrak{g})}\left(\varepsilon^{l-1}\right)\right) \eta_{L(\mathcal{g})}\left(\left[\varepsilon_{\nu}\right]^{-1}\right)$ $=\delta_{J L(\mathfrak{g})}+\eta_{L(\mathfrak{g})}(\varepsilon)+\ldots+\eta_{L(\mathfrak{g})}\left(\varepsilon^{l-1}\right)$, we have $\eta_{L(\mathfrak{g})}\left(\left[\varepsilon_{\nu}\right]\right)\left(A_{L(\mathfrak{g}) / L}\right)=A_{L(\mathfrak{g}) / L}$. Therefore

$$
\left(\eta_{L(\mathcal{g})}(\varepsilon)-\delta_{J_{L(\mathfrak{g})}}\right) a_{L(\mathfrak{g})}\left(\left[\varepsilon_{\nu}\right]^{-1}\right) \in A_{L(\mathfrak{g}) / L} \quad\left(\varepsilon_{\nu} \in G(L / K)\right) .
$$

We denote by $\eta_{L(g)}(\varepsilon)_{B_{L(g) / L}}$ the restriction of $\eta_{L(g)}(\varepsilon)$ of $B_{L(g) / L}$. Let $r$ be any prime except $p$. Then $r$-addic representation $\left\{M_{r}\left(\eta_{L(g)}\left(\varepsilon^{\nu}\right)_{B_{L(g) / L}}\right)\right\}$ of $\left\{\eta_{L(\mathfrak{g})}\left(\varepsilon^{\nu}\right)_{B_{L(\mathfrak{g}), L}}\right\}$ is equivalent to 


$$
\left(\begin{array}{lll}
\zeta^{\nu} E_{2\left(g_{L}-1\right)^{*}} & & \\
\left.\zeta^{2 \nu} E_{2\left(g^{-1}\right.}\right) & \\
& \cdot \zeta^{(l-1) \nu} E_{2\left(g_{L}-1\right)}
\end{array}\right),
$$

where $g_{L}$ is the genus of $L / k, \zeta$ is an $l$-th root of unity and $E_{2\left(g_{L}-1\right)}$ is the identity matrix of degree $2\left(g_{L}-1\right)$. This shows that

$$
\begin{aligned}
\operatorname{det} M_{r}\left(\delta_{B_{L}(\mathrm{~g}), L}-\eta_{L(\mathcal{g})}(\varepsilon)_{B_{L}(\mathrm{~g}), L}\right) & =\left(\prod_{\nu=1}^{l-1}\left(1-\zeta^{\nu}\right)\right)^{2\left(g_{L}-1\right)} \\
& =l^{2\left(g_{L}-1\right)} .
\end{aligned}
$$

We denote by $\mathfrak{h}$ the group of points $t$ of $J_{L(\mathfrak{g})}$ such that

$$
\left(\delta_{J}(\mathrm{~g})-\eta_{L(\mathrm{~g})}(\varepsilon)\right) t \in A_{L(\mathrm{~g}) / L}
$$

Then we have

$$
\begin{aligned}
I_{1}=\left[\mathfrak{h}: A_{L(\mathfrak{g}) / L}\right] & =\left[\left(\delta_{B_{L}(\mathfrak{g}) / L}-\eta_{L(\mathfrak{g})}(\varepsilon)_{B_{L(\mathcal{g}) / L}}\right)^{-1}(0):\{0\}\right] \\
& \leqq \operatorname{det} M_{r}\left(\left(\delta_{B_{L}(\mathfrak{g}) / L}-\eta_{L(\mathfrak{g})}(\varepsilon)_{B_{L(\mathfrak{g}) / L}}\right)\right) \\
& =l^{2\left(g_{L}-1\right) .}
\end{aligned}
$$

On the other hand, by virtue of Riemann's conjecture of congrunce $\zeta$-functions, the absolute values of characteristic roots of $M_{r}\left(p_{B_{L}(g) / L}\right)^{4)}$ are all $\sqrt{q}$. Hence the absolute values of characteristic roots of $M_{r}\left(\delta_{B_{L}(g) / L}-\mathfrak{p}_{B_{L}(g), L}\right)$ are not less than $\sqrt{ } q-2 \sqrt{q}+1$. This shows that

$$
\left|\operatorname{det} M_{r}\left(\delta_{B_{L}(\mathfrak{g}) / L}-\mathfrak{p}_{B_{L}(\mathfrak{g}) / L}\right)\right| \supseteq(q-2 \sqrt{q}+1)^{(i-1)\left(g_{L}-1\right)} .
$$

Since $J_{L(\mathfrak{g})}(, k)=\left(\delta_{J_{L}(\mathfrak{g})}-\mathfrak{p}_{J_{L}(\mathfrak{g})}\right)^{-1}(0), \quad A_{L(\mathfrak{g}) / L}(, k)=\left(\delta_{A_{L}(\mathfrak{g})_{i} L}-\mathfrak{p}_{A_{L}(\mathfrak{g})_{i} L}\right)^{-1}(0)$, $\left.B_{L(\mathfrak{g}) / L}(, k)=\delta_{B_{L}(\mathfrak{g}), L}-\mathfrak{p}_{B_{L}(\mathfrak{g}) / L}\right)^{-1}(0)$ and $\delta_{*}-\mathfrak{P}_{*}$ are separable, we have

$$
\begin{aligned}
I_{2} & =\left[J_{L(\mathfrak{g})}(, k): A_{L(\mathfrak{g}) / K}(, k)\right]=\operatorname{det} M_{r}\left(\delta_{J_{L}(\mathfrak{g})}-\mathfrak{p}_{J_{L}(\mathfrak{g})}\right) / \operatorname{det} M_{r}\left(\delta_{A_{L}(\mathfrak{g}) / L}-\mathfrak{p}_{A_{L}(\mathfrak{g})_{L} L}\right) \\
& =\operatorname{det} M_{r}\left(\delta_{B_{L(\mathfrak{g}) / L}}-\mathfrak{p}_{B_{L}(\mathfrak{g}) / L}\right) \geqq(q+1-2 \sqrt{q})\left(g_{L}-1\right)(l-1) .
\end{aligned}
$$

From $q \geq 11$ we have $(q+1-2 \sqrt{q})>5$. On the other hand $\log _{10} 5>\frac{2}{3}$, hence $(l-1) \log _{10} 5>\frac{2}{3}(l-1)>2 \log _{10} l$ for $l>1$. This shows that $(q+1$ $-2 \sqrt{ } q)^{(l-1)}>l^{2}$. By virtue of $g_{L}>g_{K}>1, I_{2} \geq(q+1-2 \sqrt{ } q)^{(l-1)\left(g_{L}-1\right)}>l^{2\left(g_{L}-1\right)}$ $\Rightarrow I_{1}$ for $l>1$. This proves that $\mathfrak{a}_{1}=\mathfrak{h} \cap J_{L(\mathfrak{g})}(, k)$ is a proper subgroup of $J_{L(\mathfrak{g})}(, k)$. From (4) all $a_{L(\mathfrak{g})}\left(\varepsilon^{\prime}\right)\left(\varepsilon^{\prime} \in G(L(\mathfrak{g}) / K)\right)$ belong to $g_{1}$. Hence, by

4) $p_{B_{L}(\mathrm{~g}) / L}$ means the endomorphism of $B_{L(\mathrm{~g}) / L}$ induced by the automorphism $\xi \rightarrow \xi^{q}$ of the universal domain. 
virtue of lemma 1 , it is sufficient to prove $g_{1}=\eta_{L / g)}\left(\left[\varepsilon_{\nu}\right] \varepsilon^{\mu}\right)\left(g_{1}\right) \quad\left(\varepsilon_{\nu} \in G(L / K)\right)$. Since $\zeta$ is a primitive $l$-th root of unity, $1+\zeta+\ldots+\zeta^{\nu-1}(\nu=1,2, \ldots, l-1)$ are units in $Q(\zeta)$, where $Q$ means the field of rational numbers. This shows that

$$
\left(\delta_{B_{L(\mathfrak{g}) / L}}+\eta_{L(\mathfrak{g})}(\varepsilon)_{B_{L(\mathfrak{g}) / L}}+\ldots+\eta_{L(\mathfrak{g})}\left(\varepsilon^{\nu-1}\right)_{\left.B_{L(g}\right)_{i}}\right) \quad(\nu=1,2, \ldots, l-1)
$$

are automorphisms of $B_{L(\mathfrak{g}) / L}$. On the other hand $\mathfrak{h}$ is generated by $A_{L(\mathfrak{g}) ! L}$ and $\mathfrak{h}_{B_{L}(\mathfrak{g}), L}=\left\{t \mid t \in B_{L(g) / L}, \quad\left(\delta_{B_{L}(g) / L}-\eta_{L(g)}(\varepsilon)\right) t \in A_{L(\mathfrak{g}) / L}\right\}$. Moreover we observe that

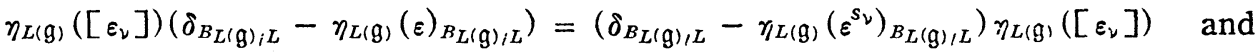
$\eta_{L(g)}\left(\left[\varepsilon_{v}\right]^{-1}\right) B_{L(g) / L}=B_{L(g) / L}$.

This shows that $\eta_{L(\mathfrak{g})}\left(\left[\varepsilon_{\nu}\right]\right)\left(\mathfrak{h}_{\left.B_{L^{\prime}} \mathfrak{g}\right) / L}\right)=\mathfrak{h}_{B_{L(\mathfrak{g}) / L}}$, namely $\mathfrak{h}=\eta_{L(\mathfrak{g})}\left(\left[\varepsilon_{v}\right]\right)(\mathfrak{h})$. Hence $\eta_{L(\mathfrak{g})}\left(\left[\varepsilon_{\nu}\right]\right)\left(g_{1}\right)=\eta_{L(\mathfrak{g})}\left(\left[\varepsilon_{\nu}\right]\right)\left(\mathfrak{h} \cap J_{L(\mathfrak{g})}(, k)\right)=\mathfrak{h} \cap J_{L(\mathfrak{g})}(, k)=\mathfrak{g}_{1}$.

\section{REFERENCE}

[1] H. Morikawa, Generalized jacobian varieties and separable abelian extension of function fields, Nagoya Journal, 12 (1957), pp. 231-254.

\section{Mathematical Institute}

Nagoya University 